\title{
HOLOMORPHIC RIEMANNIAN MAPS
}

\author{
BAYRAM ŞAHIN
}

\begin{abstract}
We introduce holomorphic Riemannian maps between almost Hermitian manifolds as a generalization of holomorphic submanifolds and holomorphic submersions, give examples and obtain a geometric characterization of harmonic holomorphic Riemannian maps from almost Hermitian manifolds to Kähler manifolds.
\end{abstract}

\section{INTRODUCTION}

In 1992, Fischer introduced Riemannian maps between Riemannian manifolds in [6] as a generalization of the notions of isometric immersions and Riemannian submersions, for Riemannian submersions, 8] and [11, see also [5] and [16. Let $F:\left(M_{1}, g_{1}\right) \longrightarrow\left(M_{2}, g_{2}\right)$ be a smooth map between Riemannian manifolds such that $0<\operatorname{rank} F<\min \{m, n\}$, where $\operatorname{dim} M_{1}=m$ and $\operatorname{dim} M_{2}=n$. Then we denote the kernel space of $F_{*}$ by $k e r F_{*}$ and consider the orthogonal complementary space $\mathcal{H}=\left(k e r F_{*}\right)^{\perp}$ to $k e r F_{*}$. Then the tangent bundle of $M_{1}$ has the following decomposition

$$
T M_{1}=k e r F_{*} \oplus \mathcal{H} .
$$

We denote the range of $F_{*}$ by range $_{*}$ and consider the orthogonal complementary space $\left(\text { range }_{*}\right)^{\perp}$ to range $_{*}$ in the tangent bundle $T M_{2}$ of $M_{2}$. Since $\operatorname{rank} F<\min \{m, n\}$, we always have $\left(\operatorname{range}_{*}\right)^{\perp}$. Thus the tangent bundle $T M_{2}$ of $M_{2}$ has the following decomposition

$$
T M_{2}=\left(\text { range }_{*}\right) \oplus\left(\text { range } F_{*}\right)^{\perp} .
$$

Now, a smooth map $F:\left(M_{1}^{m}, g_{1}\right) \longrightarrow\left(M_{2}^{n}, g_{2}\right)$ is called Riemannian map at $p_{1} \in M$ if the horizontal restriction $F_{* p_{1}}^{h}:\left(k e r F_{* p_{1}}\right)^{\perp} \longrightarrow\left(\operatorname{range}_{* p_{1}}\right)$ is a linear isometry between the inner product spaces $\left(\left(k e r F_{* p_{1}}\right)^{\perp},\left.g_{1}\left(p_{1}\right)\right|_{\left(k e r F_{* p_{1}}\right)^{\perp}}\right)$ and $\left(\right.$ range $\left.F_{* p_{1}},\left.g_{2}\left(p_{2}\right)\right|_{\left(\text {range }_{* p_{1}}\right)}\right), p_{2}=F\left(p_{1}\right)$. Therefore Fischer stated in [6] that a Riemannian map is a map which is as isometric as it can be. In another words, $F_{*}$ satisfies the equation

$$
g_{2}\left(F_{*} X, F_{*} Y\right)=g_{1}(X, Y)
$$

for $X, Y$ vector fields tangent to $\mathcal{H}$. It follows that isometric immersions and Riemannian submersions are particular Riemannian maps with $k e r F_{*}=\{0\}$ and $\left(\text { range } F_{*}\right)^{\perp}=\{0\}$. It is known that a Riemannian map is a subimmersion [6].

2010 Mathematics Subject Classification. 53C15, 53C43.

Key words and phrases. Riemannian submersion, Holomorphic submersion, holomorphic submanifold, holomorphic Riemannian map. 
In this paper, as a generalization of holomorphic submersions and holomorphic submanifolds, we introduce holomorphic Riemannian maps and investigate the harmonicity of such maps.

\section{The Gauss equation for Riemannian maps}

Let $(\bar{M}, g)$ be an almost Hermitian manifold. This means [16] that $\bar{M}$ admits a tensor field $J$ of type $(1,1)$ on $\bar{M}$ such that, $\forall X, Y \in \Gamma(T \bar{M})$, we have

$$
J^{2}=-I, \quad g(X, Y)=g(J X, J Y) .
$$

An almost Hermitian manifold $\bar{M}$ is called Kähler manifold if

$$
\left(\bar{\nabla}_{X} J\right) Y=0, \forall X, Y \in \Gamma(T \bar{M}) \text {, }
$$

where $\bar{\nabla}$ is the Levi-Civita connection on $\bar{M}$.

Let $\left(M, g_{M}\right)$ and $\left(N, g_{N}\right)$ be Riemannian manifolds and suppose that $\varphi: M \longrightarrow$ $N$ is a smooth mapping between them. Then the differential $\varphi_{*}$ of $\varphi$ can be viewed a section of the bundle $\operatorname{Hom}\left(T M, \varphi^{-1} T N\right) \longrightarrow M$, where $\varphi^{-1} T N$ is the pullback bundle which has fibres $\left(\varphi^{-1} T N\right)_{p}=T_{\varphi(p)} N, p \in M . \operatorname{Hom}\left(T M, \varphi^{-1} T N\right)$ has a connection $\nabla$ induced from the Levi-Civita connection $\nabla^{M}$ and the pullback connection $\nabla^{\varphi}$. Then the second fundamental form of $\varphi$ is given by

$$
\left(\nabla \varphi_{*}\right)(X, Y)=\nabla_{X}^{\varphi} \varphi_{*}(Y)-\varphi_{*}\left(\nabla_{X}^{M} Y\right)
$$

for $X, Y \in \Gamma(T M)$. It is known that the second fundamental form is symmetric. A smooth map $\varphi:\left(M, g_{M}\right) \longrightarrow\left(N, g_{N}\right)$ is said to be harmonic if $\operatorname{trace}\left(\nabla \varphi_{*}\right)=0$. On the other hand, the tension field of $\varphi$ is the section $\tau(\varphi)$ of $\Gamma\left(\varphi^{-1} T N\right)$ defined by

$$
\tau(\varphi)=\operatorname{div} \varphi_{*}=\sum_{i=1}^{m}\left(\nabla \varphi_{*}\right)\left(e_{i}, e_{i}\right)
$$

where $\left\{e_{1}, \ldots, e_{m}\right\}$ is the orthonormal frame on $M$. Then it follows that $\varphi$ is harmonic if and only if $\tau(\varphi)=0$.

For a Riemannian map, we have the following.

Lemma 2.1. 13 Let $F$ be a Riemannian map from a Riemannian manifold $\left(M_{1}, g_{1}\right)$ to a Riemannian manifold $\left(M_{2}, g_{2}\right)$. Then

$$
g_{2}\left(\left(\nabla F_{*}\right)(X, Y), F_{*}(Z)\right)=0, \forall X, Y, Z \in \Gamma\left(\left(k e r F_{*}\right)^{\perp}\right) .
$$

From now on, for simplicity, we denote by $\nabla^{2}$ both the Levi-Civita connection of $\left(M_{2}, g_{2}\right)$ and its pullback along $F$. Then according to [10, for any vector field $X$ on $M_{1}$ and any section $V$ of $\left(\text { range }_{*}\right)^{\perp}$, where $\left(\text { range } F_{*}\right)^{\perp}$ is the subbundle of $F^{-1}\left(T M_{2}\right)$ with fiber $\left(F_{*}\left(T_{p} M\right)\right)^{\perp}$-orthogonal complement of $F_{*}\left(T_{p} M\right)$ for $g_{2}$ over $p$, we have $\nabla_{X}^{F \perp} V$ which is the orthogonal projection of $\nabla_{X}^{2} V$ on $\left(F_{*}(T M)\right)^{\perp}$. In [10], the author also showed that $\nabla^{F \perp}$ is a linear connection on $\left(F_{*}(T M)\right)^{\perp}$ such that $\nabla^{F \perp} g_{2}=0$. We now define $\mathcal{S}_{V}$ as

$$
\nabla_{F_{*} X}^{2} V=-\mathcal{S}_{V} F_{*} X+\nabla_{X}^{F \perp} V,
$$

where $\mathcal{S}_{V} F_{*} X$ is the tangential component (a vector field along $F$ ) of $\nabla_{F_{*} X}^{2} V$. It is easy to see that $\mathcal{S}_{V} F_{*} X$ is bilinear in $V$ and $F_{*} X$ and $\mathcal{S}_{V} F_{*} X$ at $p$ depends only on $V_{p}$ and $F_{* p} X_{p}$. By direct computations, we obtain

$$
g_{2}\left(\mathcal{S}_{V} F_{*} X, F_{*} Y\right)=g_{2}\left(V,\left(\nabla F_{*}\right)(X, Y)\right),
$$


for $X, Y \in \Gamma\left(\left(k e r F_{*}\right)^{\perp}\right)$ and $V \in \Gamma\left(\left(\text { range }_{*}\right)^{\perp}\right)$. Since $\left(\nabla F_{*}\right)$ is symmetric, it follows that $\mathcal{S}_{V}$ is a symmetric linear transformation of $r a n g e F_{*}$.

By using (2.6) and (2.3) we obtain the following equation which will be called the Gauss equation for a Riemannian map between Riemannian manifolds.

Lemma 2.2. Let $F:\left(M_{1}, g_{1}\right) \longrightarrow\left(M_{2}, g_{2}\right)$ be a Riemannian map from Riemannian manifold $M_{1}$ to a Riemannian manifold $M_{2}$. Then we have

$$
g_{2}\left(R^{2}\left(F_{*} X, F_{*} Y\right) F_{*} Z, F_{*} T\right)=g_{1}\left(R^{1}(X, Y) Z, T\right)+g_{2}\left(\left(\nabla F_{*}\right)(X, Z),\left(\nabla F_{*}\right)(Y, T)\right)
$$

$$
\text { - } g_{2}\left(\left(\nabla F_{*}\right)(Y, Z),\left(\nabla F_{*}\right)(X, T)\right)
$$

for $X, Y, Z, T \in \Gamma\left(\left(k e r F_{*}\right)^{\perp}\right)$, where $R^{1}$ and $R^{2}$ denote curvature tensors of $\nabla^{1}$ and $\nabla^{2}$ which are metric connections on $M_{1}$ and $M_{2}$, respectively.

\section{Holomorphic RiemanNian maps}

In this section, we define holomorphic Riemannian maps and obtain a geometric characterization of harmonic holomorphic Riemannian maps from a Kähler manifold to an almost Hermitian manifold.

Definition 1. Let $F$ be a Riemannian map from an almost Hermitian manifold $\left(M_{1}, g_{1}, J_{1}\right)$ to an almost Hermitian manifold $\left(M_{2}, g_{2}, J_{2}\right)$. Then we say that $F$ is a holomorphic Riemannian map at $p \in M_{1}$ if

$$
J_{2} F_{*}=F_{*} J_{1} .
$$

If $F$ is a holomorphic Riemannian map at every point $p \in M_{1}$ then we say that $F$ is a holomorphic Riemannian map between $M_{1}$ and $M_{2}$.

It is known that vertical and horizontal distributions of an almost Hermitian submersion are invariant with respect to the complex structure of the total manifold. Next, we show that this is true for a holomorphic Riemannian map.

Lemma 3.1. Let $F$ be a holomorphic Riemannian map between almost Hermitian manifolds $\left(M_{1}, g_{1}, J_{1}\right)$ and $\left(M_{2}, g_{2}, J_{2}\right)$. Then the distributions $k e r F_{*}$ and $\left(k e r F_{*}\right)^{\perp}$ are invariant with respect to $J_{1}$.

Proof. For $X \in \Gamma\left(k e r F_{*}\right)$, from (3.1) we have $F_{*}\left(J_{1} X\right)=J_{2} F_{*}(X)=0$ which implies that $J_{1} X \in \Gamma\left(k e r F_{*}\right)$. In a similar way, one shows that $\left(k e r F_{*}\right)^{\perp}$ is invariant.

In a similar way, it is easy to see that $\left(\text { range }_{*}\right)^{\perp}$ is invariant under the action of $J_{2}$. We now give examples of holomorphic Riemannian maps.

Example 1. Every holomorphic submersion between almost Hermitian manifolds is a holomorphic Riemannian map with $\left(\text { range }_{*}\right)^{\perp}=\{0\}$, For holomorphic (almost Hermitian) submersions, see; [5], 15.

Example 2. Every Kählerian submanifold of a Kähler manifold is a holomorphic Riemannian map with $k e r F_{*}=\{0\}$. For Kählerian submanifolds, see; 16]. 
In the following $R^{2 m}$ denotes the Euclidean $2 m$ - space with the standart metric. An almost complex structure $J$ on $R^{2 m}$ is said to be compatible if $\left(R^{2 m}, J\right)$ is complex analytically isometric to the complex number space $C^{m}$ with the standart flat Kählerian metric. We denote by $J$ the compatible almost complex structure on $R^{2 m}$ defined by

$$
J\left(a^{1}, \ldots, a^{2 m}\right)=\left(-a^{2}, a^{1}, \ldots,-a^{2 m}, a^{2 m-1}\right) .
$$

Example 3. Consider the following Riemannian map given by

$$
F: \begin{gathered}
R^{4} \\
\left(x_{1}, x_{2}, x_{3}, x_{4}\right)
\end{gathered} \longrightarrow \quad \begin{gathered}
R^{4} \\
\left(\frac{x_{1}+x_{3}}{\sqrt{2}}, \frac{x_{2}+x_{4}}{\sqrt{2}}, 0,0\right) .
\end{gathered}
$$

Then $F$ is a holomorphic Riemannian map.

Remark 1. We note that the notion of invariant Riemannian map has been introduced in [13] as a generalization of invariant immersion of almost Hermitian manifolds and holomorphic Riemannian submersions. One can see that every holomorphic Riemannian map is an invariant Riemannian map, but the converse is not true. In other words, an invariant Riemannian map may not be a holomorphic Riemannian map.

Since $F$ is a subimmersion, it follows that the rank of $F$ is constant on $M_{1}$, then the rank theorem for functions implies that $k e r F_{*}$ is an integrable subbundle of $T M_{1}$, (1], page:205). We now investigate the harmonicity of holomorphic Riemannian maps. We first note that if $M_{1}$ and $M_{2}$ are Kähler manifolds and $F: M_{1} \longrightarrow M_{2}$ is a holomorphic map then $F$ is harmonic [2]. But there is no guarantee when $M_{1}$ or $M_{2}$ is an almost Hermitian manifold.

Theorem 3.1. Let $F$ be a holomorphic Riemannian map from a Kähler manifold $\left(M_{1}, g, J_{1}\right)$ to almost Hermitian manifold $\left(M_{2}, g_{2}, J_{2}\right)$. Then $F$ is harmonic if and only if the distribution $F_{*}\left(\left(k e r F_{*}\right)^{\perp}\right)$ is minimal.

Proof. Since $T M_{1}=k e r F_{*} \oplus\left(k e r F_{*}\right)^{\perp}$, we can write $\tau=\tau^{1}+\tau^{2}$, where $\tau^{1}$ and $\tau^{2}$ are the parts of $\tau$ in $k e r F_{*}$ and $\left(k e r F_{*}\right)^{\perp}$, respectively. First we compute $\tau^{1}=$ $\sum_{i=1}^{n_{1}}\left(\nabla F_{*}\right)\left(e_{i}, e_{i}\right)$, where $\left\{e_{1}, \ldots, e_{n_{1}}\right\}$ is a basis of $k e r F_{*}$. From (2.3), we have

$$
\tau^{1}=-\sum_{i=1}^{n_{1}} F_{*}\left(\nabla_{e_{i}}^{1} e_{i}\right)
$$

We note that, since $\left(k e r F_{*}\right)$ is an invariant space with respect to $J_{1}$, then $\left\{J_{1} e_{i}\right\}_{i=1}^{n_{1}}$ is also basis of $k e r F_{*}$. Thus we can write

$$
\tau^{1}=\sum_{i=1}^{n_{1}}\left(\nabla F_{*}\right)\left(J_{1} e_{i}, J_{1} e_{i}\right)=-\sum_{i=1}^{n_{1}} F_{*}\left(\nabla_{J_{1} e_{i}}^{1} J_{1} e_{i}\right) .
$$

Since $M_{1}$ is a Kähler manifold and $k e r F_{*}$ is integrable, using (3.1), we obtain

$$
\tau^{1}=-\sum_{i=1}^{n_{1}} J_{2} F_{*}\left(\nabla_{e_{i}}^{1} J_{1} e_{i}\right)
$$

Using again (3.1), we derive

$$
\tau^{1}=\sum_{i=1}^{n_{1}} F_{*}\left(\nabla_{e_{i}}^{1} e_{i}\right)
$$


Thus (3.2) and (3.3) imply that $\tau^{1}=0$. On the other hand, using Lemma 2.1 and (2.3) we obtain

$$
\tau^{2}=g_{2}\left(\sum_{s=1}^{m_{2}} \sum_{a=1}^{n_{1}}\left(\nabla_{e_{a}}^{F} F_{*}\left(e_{a}\right), \mu_{s}\right) \mu_{s}=H_{\left(\text {range }_{*}\right)},\right.
$$

where $H_{\left(\text {range }_{*}\right)}$ is the mean curvature vector field of $\left(\right.$ range $\left.F_{*}\right)$. Then our assertion follows from above equation and (3.3).

Next, by using (2.1) and (2.2) we have the following.

Lemma 3.2. Let $F$ be a holomorphic Riemannian map from an almost Hermitian manifold $\left(M_{1}, g_{1}, J_{1}\right)$ to a Kähler manifold $\left(M_{2}, g_{2}, J_{2}\right)$. Then we have

$$
\left(\nabla F_{*}\right)\left(X, J_{1} Y\right)=\left(\nabla F_{*}\right)\left(Y, J_{1} X\right)=J_{2}\left(\nabla F_{*}\right)(X, Y),
$$

for $X, Y \in \Gamma\left(\left(k e r F_{*}\right)^{\perp}\right)$.

Lemma 3.3. Let $F$ be a holomorphic Riemannian map from an almost Hermitian manifold $\left(M_{1}, g_{1}, J_{1}\right)$ to a Kähler manifold $\left(M_{2}, g_{2}, J_{2}\right)$. Then we have

$$
\begin{aligned}
g_{1}\left(R^{1}\left(X, J_{1} X\right) J_{1} X, X\right) & =g_{2}\left(R^{2}\left(F_{*} X, J_{2} F_{*} X\right) J_{2} F_{*} X, F_{*} X\right) \\
& -2\left\|\left(\nabla F_{*}\right)(X, X)\right\|^{2}
\end{aligned}
$$

for $X \in \Gamma\left(\left(k e r F_{*}\right)^{\perp}\right)$.

Proof. Putting $Y=J_{1} X, Z=J_{1} X$ and $T=X$ in (2.8) and by using (3.1) and (3.4) we obtain (3.5).

As a result of Lemma 3.3, we have the following result for the leaves of $\left(k e r F_{*}\right)^{\perp}$.

Theorem 3.2. Let $F$ be a holomorphic Riemannian map from an almost Hermitian manifold $\left(M_{1}, g_{1}, J_{1}\right)$ to a complex space form $\left(M_{2}(c), g_{2}, J_{2}\right)$ of constant holomorphic sectional curvature $c$ such that $\left(k e r F_{*}\right)^{\perp}$ is integrable. Then the integral manifold of $\left(k e r F_{*}\right)^{\perp}$ is a complex space form $M^{\prime}(c)$ if and only if $\left(\nabla F_{*}\right)(X, X)=0$ for $X \in \Gamma\left(\left(k e r F_{*}\right)^{\perp}\right)$.

Concluding Remarks. It is known that the complex techniques in relativity have been very effective tools for understanding spacetime geometry [9]. Indeed, complex manifolds have two interesting classes of Kähler manifolds. One is Calabi-Yau manifolds which have their applications in superstring theory [3]. The other one is Teichmuler spaces applicable to relativity [14. It is also important to note that CR-structures have been extensively used in spacetime geometry of relativity [12]. For complex methods in general relativity, see: 4 .

In [6], Fischer proposed an approach to build a quantum model and he pointed out the success of such a program of building a quantum model of nature using Riemannian maps would provide an interesting relationship between Riemannian maps, harmonic maps and Lagrangian field theory on the mathematical side, and Maxwells equation, Schrödingers equation and their proposed generalization on the physical side. It is also important to note that Riemannian maps satisfy the Eikonal equation which is a bridge between geometric optics and physical optics. For Riemannian maps and their applications in spacetime geometry, see [7. As a unification of Riemannian maps and complex geometry, holomorphic Riemannian maps may have their applications in mathematical physics and physical optics. 


\section{REFERENCES}

[1] Abraham,R., Marsden, J.E., Ratiu,T., Manifolds, Tensor Analysis, and Applications, Springer-Verlag, Newyork, 1988.

[2] Baird,P. and Wood, J. C., Harmonic morphisms between Riemannian manifolds, Clarendon press, Oxford, (2003).

[3] Candelas, P., Horowitz, G., Strominger, A., Witten, E., Vacuum configurations for superstrings, Nucl. Phys B, 258, (1985), 46-74.

[4] Esposito, G., From spinor geometry to complex general relativity, International Journal of Geometric Methods in Modern Physics, Vol. 2, No. 4 (2005), 675-731

[5] Falcitelli, M., Ianus, S., Pastore, A. M., Riemannian submersions and related topics. World Scientific Publishing Co., Inc., River Edge, NJ, (2004).

[6] Fischer, A. E.: Riemannian maps between Riemannian manifolds, Contemporary math. 132, 331-366, (1992).

[7] Garcia-Rio, E., Kupeli, D.N., Semi-Riemannian maps and Their Applications, Kluwer Academic, (1999).

[8] Gray, A., Pseudo-Riemannian almost product manifolds and submersions. J. Math. Mech. 16, 715-737, (1967).

[9] Lerner, D.E., Sommers, P.D., Complex manifold techniques in theoretical physics Pitman Advanced Publishing, (1979).

[10] Nore, T., Second fundamental form of a map, Ann. Mat. Pur. and Appl., 146, (1987), 281-310.

[11] O'Neill, B., The fundamental equations of a submersion, Michigan Math. J. 13, (1966), 459469.

[12] Penrose, R., Physical spacetime and nonrealizable CR-structure, vol:39, Proc. Smposia in Pure Math, (1983), 401-422.

[13] Șahin,B., Invariant and anti-invariant Riemannian maps to Kähler manifolds, Int. J. Geom. Methods Mod. Phys., vol:7, no:3 (2010), 1-19.

[14] Tromba, A.J., Teichmuller Theory in Riemannian Geometry, Birkhauser Verlag, Boston, 1992.

[15] Watson, B., Almost Hermitian submersion, J. Differential Geometry, 11, (1976), 147-165.

[16] Yano, K. and Kon, M., Structures on manifolds, Ser. Pure Math. World Scientific,(1984). 